\title{
SOME ADDITIONAL CONSIDERATIONS ON THE METHOD OF GENETICAL ANALYSIS FOR INDUCED CONTINUOUS VARIATION OF SELF-FERTILISING PLANTS
}

\author{
K. YONEZAWA* \\ Department of Genetics, University of Birmingham, Birmingham B/5 2TT
}

Received 8.v.79

\section{SUMMARY}

\begin{abstract}
Genetic parameters for describing the induced continuous variation of selffertilising plants have been defined in terms of mutation rate per allele and the additive and dominance effects of the genes concerned. Estimation and interpretation of these parameters are discussed.

It is shown that no single generation up to the $\mathrm{M}_{3}$ provides enough statistics to estimate the parameters, unless mutagenic treatment is applied to zygotic cells. As in the case of generations derived from hybridisation between two inbred lines, information on the average degree of dominance and gene association in parental lines can be obtained using these estimates. The mutation rate can be estimated when two parental lines with significantly different phenotypic values are investigated simultaneously.
\end{abstract}

\section{INTRODUCTION}

WHILE many experiments have been conducted to assess the amount of induced genetic variation in self-fertilising plants, the genetical interpretation of the variation has received relatively less attention. Further advance awaits assessment of the biometrical method for manipulating induced variation.

Diallel crosses have been tried by some workers (e.g. Lawrence, 1965) using inbred lines which have been selected from mutagenically treated populations. The limitation of this method, however, is the time required to produce the pure lines, and in some plant species, the difficulty of making a sufficiently large number of cross combinations and $F_{1}$ seeds per cross. Furthermore, selected lines are not suitable for investigating the original genetic effects of mutagens, and they may not meet the assumption of uncorrelated gene distributions (Jinks, 1954), which may cause misinterpretation of the results of diallel crosses.

Aastveit and Gault (1967) have suggested a simpler method by which genetic variances and covariances of the $\mathrm{M}_{2}, \mathrm{M}_{3}$ and $\mathrm{M}_{4}$ populations are partitioned into the components which more directly reflect the genetic effects of individual genes. In this method, all of the $M_{2}, M_{3}$ and $M_{4}$ populations have to be grown in the same experiment in the same year to eliminate any bias due to genotype $\times$ environment interaction.

Noticing this inconvenience, Virk et al. (1978) have proposed another method which requires only a single generation of a population with a hierarchical pedigree structure. As will be explained later, however, their idea is not applicable before the $\mathrm{M}_{4}$ generation unless the mutagenic treat-

* Permanent address: Laboratory of Plant Breeding, Faculty of Agriculture, Kyoto University, Kyoto 606, Japan.

$43 / 2-c$ 
ment is applied to zygotic cells or at a very early stage of embryo development. Neither does this method provide an estimate of the multiplicative component of the additive dominance gene effects, which is necessary not only in predicting the efficiency of artificial selection but also in obtaining some information about the association of dominant genes in the parental lines used.

In this paper a number of methods which are practicable up to the $M_{3}$ generation, will be discussed, since the $\mathbf{M}_{3}$ is regarded as being the best generation to start selection for breeding (Yonezawa, 1975). Interpretation of the estimates of the parameters will also be discussed.

\section{Genetic Structure of THE $\mathrm{M}_{1}$ AND $\mathrm{M}_{2}$ POpulations}

$\mathrm{M}_{1}$ plants are in general multicellular at the time of mutagenic treatment, mutations occurring independently in each of the constituent cells. For instance, in cereal crops such as rice and wheat, embryos in mature seeds are well differentiated and carry a number of multicellular spike primordia (e.g. Osone, 1963). This means that $\mathbf{M}_{1}$ plants are genetically chimeric at the levels of both spike and whole plant, and therefore, the genetical segregation in $\mathrm{M}_{1}$ spike and plant progenies is different from that in the progeny of $F_{1}$ individuals.

Now, let us consider a single gene locus at which the parental allele $G$ mutates to mutant allele $g$, the mutation rate of $G \rightarrow g$ being $m$. Then, assuming independent occurrence of mutations, the expected frequencies of $G G, G g$ and $g g$ cells in mutagenically treated embryos are

$$
(1-m)^{2}, \quad 2 m(1-m) \text { and } m^{2}
$$

respectively. Since mutation rate $m$ is of the order of $10^{-3}$ or lower (Yonezawa and Yamagata, 1977), these values can be approximated by $1-2 m, 2 m$ and zero, respectively. So, if $\mathbf{M}_{\mathbf{1}}$ spike primordia consist of $C$ initial cells at the time of mutagenic treatment, the frequency of the primordia which are of the $G g$ genotype for $i$ cells among the $C$ is given by

$$
\left(\begin{array}{c}
C \\
i
\end{array}\right)(2 m)^{i}(1-2 m)^{C-i} \text {. }
$$

Since the number $C$ is much smaller than $\frac{x}{m}$, this formula can be approximated by

$$
\left\{\begin{array}{lll}
1-2 C m & \ldots & i=0 \\
2 C m & \ldots & i=1 \\
0 & \ldots & i \geqq 2 .
\end{array}\right.
$$

Hence, for this locus, there is no segregation in the $1-2 \mathrm{Cm}$ of the total $\mathrm{M}_{1}$ spiked-derived $\mathrm{M}_{2}$ lines, and the remaining $2 \mathrm{Cm}$ lines segregate with the ratio

$$
\frac{4 C-3}{4 C} G G: \frac{1}{2 C} G g: \frac{1}{4 C} g g,
$$

which indicates that the ratio $\frac{1}{4}: \frac{1}{2}: \frac{1}{4}$ as assumed by Virk et al. (1978) does not hold unless $C=1$. If $C$ is made equal to the total number of the initial cells contained in an embryo, the above ratio represents the segregation in $\mathbf{M}_{1}$-plant-derived $\mathbf{M}_{2}$ lines. 
Genotypic frequencies of the $\mathbf{M}_{2}$ population as a whole are independent of the cell number, and are given by

$$
\left(1-\frac{3}{2} m\right) G G: m G g: \frac{1}{2} m g g \text {. }
$$

The expected genotypic frequencies in various pedigree units (lines, families or population as a whole) in the following generations are readily obtained by the above ratios.

With $k$ genes concerned with the character at issue, the number of mutations occurring simultaneously in a single cell is expected to be subject to the Poisson distribution with mean $2 \mathrm{~km}$, which is likely to be smaller than unity. This is another characteristic of induced variation, that mutant individuals carrying two or more mutant genes will be very infrequent, indicating that effects of linkage, if any, would be much less than in genetic variation caused by hybridisation.

\section{Definition AND Estimation of Genetic PARAmeters}

Means and genetic variances of the populations following mutagenic treatment are formulated below in terms of the genetic parameters to be estimated.

Using the additive-dominance model (Mather and Jinks, 1971) and the genotypic frequencies formulated in the preceding section, the mean and genetic variance of $M_{2}$ populations are described as

$$
\bar{M}_{2}=\bar{M}_{0}-2[d]_{m}+[h]_{m},
$$

and, by omitting the terms with the second power of the mutation rate $m$,

respectively, where

$$
V_{M_{2}}=3 D_{m}+H_{m}-2 F_{m}
$$

$$
\begin{aligned}
\bar{M}_{0} & =\text { mean value of the parent used, } \\
{[d]_{m} } & =\Sigma \pm m d, \quad(d \geqq 0) \\
{[h]_{m} } & =\Sigma m h, \\
D_{m} & =\Sigma m d^{2} \\
H_{m} & =\Sigma m h^{2} \\
F_{m} & =\Sigma \pm m d h
\end{aligned}
$$

The symbols $d$ and $h$ are similarly defined as in Mather and Jinks (1971). The plus and minus signs in the summation of $[d]_{m}$ and $F_{m}$ correspond to whether the original alleles of the parent have larger or smaller effects than the respective mutant alleles. The summation in this case is over all the $k$ genes affecting the character at issue.

Where the $\mathbf{M}_{2}$ population is made up of the $\mathbf{M}_{2}$ lines derived from different $M_{1}$ plants or spikes, the variance $V_{M_{2}}$ can be partitioned into two statistically independent components, i.e. between and within $M_{2}$ line variances, which are

$$
V_{M_{2}}=\frac{2}{C} D_{m}+\frac{1}{2 C} H_{m}-\frac{2}{C} F_{m}
$$


and

$$
\bar{V}_{M_{2}}=\left(3-\frac{2}{C}\right) D_{m}+\left(1-\frac{1}{2 C}\right) H_{m}-2\left(1-\frac{1}{C}\right) F_{m}
$$

respectively.

The corresponding statistics of the $M_{3}$ population are formulated by

and

$$
\bar{M}_{3}=\bar{M}_{0}-2[d]_{m}+\frac{1}{2}[h]_{m},
$$

$$
V_{M_{3}}=\frac{7}{2} D_{m}+\frac{1}{2} H_{m}-F_{m} \text {. }
$$

When the $M_{3}$ population consists of the $M_{3}$ lines derived from different $M_{2}$ plants, $V_{M_{3}}$ is divided into

and

$$
V_{M_{3}}=3 D_{m}+\frac{1}{4} H_{m}-F_{m},
$$

$$
\bar{V}_{M_{3}}=\frac{1}{2} D_{m}+\frac{1}{4} H_{m}
$$

If the $M_{3}$ population is hierarchically structured by $M_{1}$ plants, the variance $V_{M_{3}}$ can be further partitioned into the two components, i.e. the variance between $M_{3}$ groups derived from different $M_{1}$ plants and the variance between $M_{3}$ lines from the same $M_{1}$ plants, which are presented by

$$
V_{\bar{M}_{3 B}}=\frac{2}{C} D_{m}+\frac{1}{8 C} H_{m}-\frac{1}{C} F_{m}
$$

and

$$
V_{\bar{M}_{3 W}}=\left(3-\frac{2}{C}\right) D_{m}+\left(\frac{1}{4}-\frac{1}{8 C}\right) H_{m}-\left(1-\frac{1}{C}\right) F_{m},
$$

respectively.

Covariance between the $\mathrm{M}_{2}$ and $\mathrm{M}_{3}$ generations may provide additional sources for parameter estimation. The genetic covariance between $\mathrm{M}_{2}$ plants and their $\mathrm{M}_{3}$ progeny means is given by

$$
W_{M_{3} / M_{2}}=3 D_{m}+\frac{1}{2} H_{m}-\frac{3}{2} F_{m},
$$

and that between $M_{2}$ lines and $M_{3}$ groups is

$$
W_{M_{3} / M_{2}}=\frac{2}{C} D_{m}+\frac{1}{4 C} H_{m}-\frac{3}{2 C} F_{m} \text {. }
$$

By using the $M_{2}$ and $M_{3}$ populations, we have two sources for the estimation of the two first-degree parameters, $[d]_{m}$ and $[h]_{m}$. Theoretically, seven sources $V_{\bar{M}_{2}}, \bar{V}_{M_{2}}, V_{\bar{M}_{3 B}}, V_{\bar{M}_{3} W}, \bar{V}_{M_{3}}, W_{\bar{M}_{3} / M_{2}}$ and $W_{\bar{M}_{3} / M_{2}}$ are available for the three second-degree parameters, $D_{m}, H_{m}$ and $F_{m}$.

Among these seven, however, those statistics that are based on the hierarchical pedigree structure originating from $\mathrm{M}_{1}$ spikes or plants, namely $V_{\bar{M}_{2}}, \bar{V}_{M_{2}}, V_{\bar{M}_{3 B}}, V_{\bar{M}_{3} W}$, and $W_{\bar{M}_{3} / M_{2}}$, include the cell number $C$ and therefore should not be used unless the value of $C$ is precisely known in advance. In practice, $C$ appears to differ with $\mathrm{M}_{1}$ spikes (Sarvella et al., 1962), and its estimate is influenced by the haplontic and diplontic selection against mutations (Yonezawa and Yamagata, 1975). The covariance $\boldsymbol{W}_{\boldsymbol{M}_{\mathbf{3}} / \mathbf{M}_{\mathbf{2}}}$ would also be unsuitable; its estimate may be much biased by both measure- 
ment error and genotype $\times$ year interaction, since $\mathrm{M}_{2}$ plants can neither be replicated nor grown in the same year as their $\mathrm{M}_{3}$ progenies.

Hence, only the three variances $V_{M_{2}}, V_{M_{3}}$ and $\bar{V}_{M_{3}}$ are reliable sources of estimates of the three second-degree parameters. Procedures for calculating these variances are outlined in table 1 assuming that the parental, $\mathbf{M}_{2}$ and $\mathbf{M}_{3}$ lines are grown with a randomised block design. Table 2 shows an estimation method for the case where the parental and $\mathbf{M}_{2}$ populations are derived from randomly selected seeds of their preceding populations. Then, applying the perfect fit solution, the estimates of the parameters are obtained by

$$
\begin{aligned}
{[\hat{d}]_{m} } & =\frac{1}{2}\left(\hat{\bar{M}}_{0}+\hat{\bar{M}}_{2}-2 \hat{M}_{3}\right), \\
{[\hat{h}]_{m} } & =2\left(\hat{M}_{2}-\hat{\bar{M}}_{3}\right), \\
\hat{D}_{m} & =-\frac{1}{4} \hat{V}_{M_{2}}+\frac{1}{2} \hat{V}_{M_{3}}+\frac{1}{2} \hat{\bar{V}}_{M_{3}} \\
\hat{H}_{m} & =\frac{1}{2} \hat{V}_{M_{2}}-\hat{V}_{M_{3}}+3 \hat{\bar{V}}_{M_{3}}, \\
\hat{F}_{m} & =-\frac{5}{8} \hat{V}_{M_{2}}+\frac{1}{4} \hat{V}_{M_{3}}+\frac{9}{4} \hat{V}_{M_{3}},
\end{aligned}
$$

where $\bar{M}_{0}, \bar{M}_{2}$ and $\bar{M}_{3}$ are of course estimated by the total mean of the respective populations.

TABLE I

A variance analysis for estimating genetic and environmental variance components of the parental, $\mathrm{M}_{2}$ and $\mathrm{M}_{3}$ populations

\begin{tabular}{lclc}
\multicolumn{1}{r}{ Item } & d.f. & M.S. & \multicolumn{1}{c}{ E.M.S. } \\
Blocks & $r-1$ & $M S_{B_{1}}$ & $\sigma_{w}^{2}+b \sigma_{B X L}^{2}+a b \sigma_{B 1}^{2}$ \\
Lines* & $a-1$ & $M S_{L}$ & $\sigma_{w}^{2}+b b \sigma_{B X L}^{2}+b r \sigma_{L}^{2}$ \\
Block $\times$ line & $(a-1)(r-1)$ & $M S_{B X L}$ & $\sigma_{w}^{2}+b \sigma_{B X L}^{2}$ \\
Within line & $a r(b-1)$ & $M S_{W}$ & $\sigma_{w}^{2}$
\end{tabular}

Estimators of the variance components;

$$
\begin{aligned}
\hat{\sigma}_{w}^{2} & =\left\{\begin{array}{lll}
M S_{W} & \ldots & \text { Significant } \\
M S_{W}^{\prime} \dagger & \ldots & \text { Non-significant }
\end{array}\right. \\
\hat{\sigma}_{L}^{2} & =\left\{\begin{array}{lll}
\left(M S_{L}-M S_{B X L}\right) / b r & \ldots & \text { Significant } \\
\left(M S_{L}-M S_{W}\right) / b r & \cdots & \text { Non-significant }
\end{array}\right.
\end{aligned}
$$

Contents of the variance components:

$\begin{array}{lcc} & \sigma_{w}^{2} & \sigma_{L}^{2} \\ \text { Parent } & E_{1+}^{+} & E_{2} \\ M_{2} \S & E_{1}+\bar{V}_{M_{2}} & E_{2}+V_{M_{2}} \\ M_{3} & E_{1}+\bar{V}_{M_{3}} & E_{2}+V_{M_{3}}\end{array}$

* Derived from different parental, $M_{1}$ and $M_{2}$ individuals for the parental, $M_{2}$ and $M_{3}$ populations respectively.

$\dagger$ Pooled mean square over the items of block $\times$ line and within line.

$\pm E_{1}$ and $E_{2}$ are the environmental variance components for within and between plots (Mather and Jinks, 1971).

$\S V_{M_{2}}$ is estimated by the summation of $\hat{\bar{V}}_{M_{2}}$ and $\hat{V}_{M_{3}}$. 


\section{TABLE 2}

A variance analysis for the parental and $\mathbf{M}_{2}$ populations derived from randomly sampled seeds

\begin{tabular}{lcll}
\multicolumn{1}{c}{ Item } & d.f. & M.S. & \multicolumn{1}{c}{ E.M.S. } \\
Blocks & $r-1$ & $M S_{B_{1}}$ & $\sigma_{w}^{2}+b \sigma_{p}^{2}+a b \sigma_{B 1}^{2}$ \\
Plots within block & $r(a-1)$ & $M S_{p}$ & $\sigma_{w w}^{2}+b \sigma_{p}^{2}$ \\
Within plot & $a r(b-1)$ & $M S_{w}$ & $\sigma_{w}^{2}$
\end{tabular}

Estimators of the variance components;

$$
\begin{aligned}
\hat{\sigma}_{w}^{2} & =\left\{\begin{array}{lll}
M S_{w} & \ldots & \sigma_{v}^{2} \\
M S_{w}^{\prime \prime}{ }^{*} & \ldots & \text { Significant } \\
\text { Non-significant }
\end{array}\right. \\
\hat{\sigma}^{2} & =\left\{\begin{array}{cll}
\left(M S_{p}-M S_{w}\right) / b & \ldots & \text { Significant } \\
0 & \ldots & \text { Non-significant }
\end{array}\right.
\end{aligned}
$$

Contents of the variance components;

$\begin{array}{lcc} & \sigma_{w}^{2} & \sigma_{p}^{2} \\ \text { Parent } & E_{1} & E_{2} \\ M_{2} & E_{1}+V_{M_{2}} & E_{2}\end{array}$

* Pooled mean square over the items of plots within block and within plot.

The variances of the above estimates are readily derived using the relationships

$$
\hat{V}\left(\hat{M}_{n}\right)=\frac{\hat{\sigma}_{w^{2}}}{a b r}+\frac{\hat{\sigma}_{B X L}^{2}}{a r}+\frac{\hat{\sigma}_{B I}^{2}}{b}+\frac{\hat{\sigma}_{L}^{2}}{a}, \quad(n=0,2,3),
$$

and

$$
\begin{gathered}
V\left(\hat{\sigma}_{w^{2}}\right)=\frac{2\left(M S_{w}\right)^{2}}{\operatorname{ar}(b-1)+2}, \\
V\left(\sigma_{L}^{2}\right)=\frac{2}{(b r)^{2}}\left(\frac{\left(M S_{L}\right)^{2}}{a+1}+\frac{\left(M S_{B X L}\right)^{2}}{(a-1)(r-1)+2}\right),
\end{gathered}
$$

which hold for any of the three populations arranged by the design of table 1. Reduction of $\hat{V}\left(\hat{\sigma}_{w^{2}}\right)$ of the parental population from those of the $M_{2}$ and $M_{3}$ population gives the variances of the estimates $\hat{V}_{M_{2}}$ and $\hat{V}_{M_{3}}$, the same reduction for $\hat{V}\left(\hat{\sigma}_{L} 2\right)$ giving the variances of $\hat{V}_{\bar{M}_{2}}$ and $\hat{V}_{\bar{M}_{3}}$. This procedure applies also the the design of table 2. But in this case, the variance of $\hat{\bar{M}}_{n}(n=0,2)$ must be modified as

$$
\hat{\nabla}\left(\hat{\bar{M}}_{n}\right)=\frac{\hat{\sigma}_{w^{2}}}{a b r}+\frac{\hat{\sigma}_{p^{2}}}{a r}+\frac{\hat{\sigma}_{B I^{2}}}{r}
$$

and $V\left(\hat{\sigma}_{L}^{2}\right)$ does not need to be calculated since the variance of the estimate of the total genetic variance $V_{M_{2}}$ can be obtained directly by the difference of $\nabla\left(\hat{\sigma}_{w}^{2}\right)$ 's.

In the case where the $\mathrm{M}_{4}$ and later populations are also available some least square method (e.g. Mather, 1949) or maximum likelihood method (e.g. Hayman, 1960) can be used, which will increase the reliability of the estimates and provide a test of the goodness-of-fit of the model. These populations should be made up of hierarchial pedigrees originating from 
$\mathbf{M}_{2}$ plants, because sampling errors in the estimates of the parameters will be minimised by this procedure and the amount of information will be increased. The method of Virk et al. (1978) is applicable in thise case, their mathematical formulae being modified to fit the above system of pedigree (they assumed a hierarchical system starting from $\mathbf{M}_{1}$ plants). For future convenience, the variances and covariances of different generations and ranks are formulated in table 3 in terms of the parameters $D_{m}, H_{m}$ and $F_{m}$. Mean of the $M_{n}$ population is given by

$$
\bar{M}_{n}=\bar{M}_{0}-2[d]_{m}+\left(\frac{1}{2}\right)^{n-2} \cdot[h]_{m} .
$$

TABLE 3

Components of genetic variances of the $\mathbf{M}_{n}$ population and covariances between the $\mathbf{M}_{n-1}$ and $\mathbf{M}_{n}$ populations

Variances;

$$
D_{m} \quad H_{m} \quad F_{m} \quad \text { Pedigree system }
$$

\begin{tabular}{|c|c|c|c|c|}
\hline $\begin{array}{l}M_{n} \text { population } \\
\text { as a whole }\end{array}$ & $4-\left(\frac{1}{2}\right)^{n-2}$ & $\left(\frac{1}{2}\right)^{n-2}$ & $-\left(\frac{1}{2}\right)^{n-3}$ & $(n \geqq 2)$ \\
\hline $\begin{array}{l}\text { Between } M_{n} \\
\quad \text { families of rank } 0\end{array}$ & 3 & $\left(\frac{1}{2}\right)^{2(n-2)}$ & $-\left(\frac{1}{2}\right)^{n-3}$ & \\
\hline $\begin{array}{l}\text { Within } M_{n} \text { family } \\
\text { of rank } 0\end{array}$ & $1-\left(\frac{1}{2}\right)^{n-2}$ & $\left(\frac{1}{2}\right)^{n-2}-\left(\frac{1}{2}\right)^{2(n-2)}$ & & $\begin{array}{l}\text { Hierarchical* } \\
(n \geqq 2)\end{array}$ \\
\hline $\begin{array}{l}\text { Between } M_{n} \text { families } \\
\text { of rank } r\end{array}$ & $\left(\frac{1}{2}\right)^{\prime}$ & $\left(\frac{1}{2}\right)^{2 n-1-3}$ & & \\
\hline $\begin{array}{l}\text { Between } M_{n} \text { lines } \\
\text { Within } M_{n} \text { line }\end{array}$ & $\begin{array}{r}4-\left(\frac{1}{2}\right)^{n-3} \\
\left(\frac{1}{2}\right)^{n-2}\end{array}$ & $\begin{array}{l}\left(\frac{1}{2}\right)^{n-1} \\
\left(\frac{1}{2}\right)^{n-1}\end{array}$ & $-\left(\frac{1}{2}\right)^{n-3}$ & $\begin{array}{l}\text { Non-hierarchical } \dagger \\
(n \geqq 3)\end{array}$ \\
\hline
\end{tabular}

Covariances;

$\mathrm{M}_{n-1}$ and $M_{n}$ families of rank 03

$\left(\frac{1}{2}\right)^{2 n-5}$

$M_{n-1}$ and $M_{n}$ families of rank $r\left(\frac{1}{2}\right)^{r}$

$\left(\frac{1}{2}\right)^{2 n-2 r-3}$ $-3\left(\frac{1}{2}\right)^{n-2}\left\{\begin{array}{l}\text { Hierarchical } \\ (n \geqq 3)\end{array}\right.$

$M_{n-1}$ individuals and their $M_{n}$ progenies

$$
4-\left(\frac{1}{2}\right)^{n-3} \quad\left(\frac{1}{2}\right)^{n-2}
$$$$
-3\left(\frac{1}{2}\right)^{n-2}
$$

Non-hierachical $(n \geqq 3)$

* The rank $r$ starts from the $M_{2}$ generation, assuming 0 for between $M_{2}$ plants or their progeny families (cf. Virk et al. (1978) for the rank notation).

$\dagger$ Applicable whether the $M_{n}$ population is hierarchially structured or not, but should be used for the latter case, e.g. for the case where the $M_{2} \sim M_{n-1}$ populations have been kept by bulk seed sampling.

\section{CRossing in the $M_{1}$ AND $M_{2}$ Generation}

Some populations which are made through hybridisation between parent, $M_{1}$ and $M_{2}$ genotypes were expected to provide additional sources for the parameter estimation. The use of some of these populations will be discussed below. 
(i) Backcrosses and pairwise crosses of $M_{1}$ and $M_{2}$ plants

In the case where $M_{1}$ plants are backcrossed to the parental line, the mean and variance of the progeny population, say $\mathbf{M}_{2}^{\prime}$ populations are represented by

and

$$
\bar{M}_{2}^{\prime}=M_{0}-[d]_{m}+[h]_{m},
$$

$$
V_{M_{2}^{\prime}}=D_{m}+H_{m}-2 F_{m}
$$

respectively. This variance may be divided into

$$
\begin{aligned}
& V_{\bar{M}_{2}^{\prime}}=\frac{1}{2 C} D_{m}+\frac{1}{2 C} H_{m}-\frac{1}{C} F_{m}=\frac{1}{2 C} V_{M_{2}^{\prime}}, \\
& \bar{V}_{M_{2}^{\prime}}=\left(1-\frac{1}{2 C}\right) V_{M_{2}^{\prime}}
\end{aligned}
$$

which are of course the between and within variance components of the $\mathbf{M}_{2}^{\prime}$ families.

For the population, say $M_{2}^{\prime \prime}$ population, which is made by the pairwise crosses between randomly selected $M_{1}$ plants, the corresponding statistics are formulated by

$$
\bar{M}_{2}^{\prime \prime}=M_{0}-2[d]_{m}+2[h]_{m}
$$

and

$$
V_{M_{2}^{\prime \prime}}=2 D_{m}+2 H_{m}-4 F_{m}=2 V_{M_{2}^{\prime}},
$$

which may be partitioned into the two components,

$$
\begin{aligned}
& V_{M_{2}^{\prime \prime}}=\frac{1}{C} D_{m}-\frac{1}{C} H_{m}-\frac{2}{C} F_{m}=2 V_{M_{2}^{\prime}}, \\
& \bar{V}_{M_{2}^{\prime \prime}}=2 \bar{V}_{M_{2}^{\prime}} .
\end{aligned}
$$

It is known from the above formulae that the $\mathbf{M}_{2}^{\prime}$ population adds one source for the estimation of the first-degree parameters, and one reliable source, i.e. $V_{M_{2}^{\prime}}$, for the second-degree parameters. The between and within $\mathrm{M}_{2}^{\prime}$ family variance components $\left(V_{\bar{M}_{2}^{\prime}}\right.$ and $\left.\bar{V}_{M_{2}^{\prime}}\right)$ cannot be used, since both of them carry the cell number $C$ and are mathematically homogeneous with the total genetic variance $V_{M_{2}^{\prime}}$. Pairwise crosses provide no new information; mean and variances of the $\mathbf{M}_{2}^{\prime \prime}$ population are homogeneous with those of the $\mathbf{M}_{2}^{\prime}$ population. All other methods including diallel crosses among $M_{1}$ plants may be difficult to carry out on a sufficiently large scale due to the physiological damage of $\mathrm{M}_{1}$ plants, and even if practicable, they add no more than the above methods.

Thus, only two statistics for each of the first- and second-degree parameters, namely, $\mathbf{M}_{\mathbf{2}}$ and $\mathbf{M}_{2}^{\prime}$ for the former parameters and $V_{\boldsymbol{M}_{2}}$ and $V_{\boldsymbol{M}_{2}^{\prime}}$ for the latter, can be used within two generations following mutagenic treatment. Hence, there is no way of estimating $D_{m}, H_{m}$ and $F_{m}$ within these two generations. 
Backcrosses between the parental line and $\mathrm{M}_{2}$ plants give the population, say $\mathrm{M}_{3}^{\prime}$ population, the statistics of which are represented by

$$
\begin{aligned}
\bar{M}_{3}^{\prime} & =\bar{M}_{0}-[d]_{m}+[h]_{m}, \\
V_{M_{3}^{\prime}} & =\frac{3}{4} D_{m}+\frac{5}{4} H_{m}-\frac{3}{2} F_{m}, \\
\bar{V}_{M_{3}^{\prime}} & =\frac{1}{4} D_{m}+\frac{1}{4} H_{m}-\frac{1}{2} F_{m},
\end{aligned}
$$

For the population (say $\mathbf{M}_{3}^{\prime \prime}$ population) which is made by pairwise crosses between $\mathrm{M}_{2}$ plants, the corresponding statistics are

$$
\begin{aligned}
\bar{M}_{3}^{\prime \prime} & =\bar{M}_{0}-2[d]+[h]_{m}=\bar{M}_{2}, \\
V_{M_{3}^{\prime \prime}} & =\frac{3}{2} D_{m}+\frac{3}{2} H_{m}-F_{m} \\
\bar{V}_{M_{3}^{\prime \prime}} & =\frac{1}{2} D_{m}+\frac{1}{2} H_{m}-F_{m}=2 \bar{V}_{M_{3}^{\prime}}
\end{aligned}
$$

The $\mathbf{M}_{3}^{\prime}$ population provides one source for the estimation of the firstdegree parameters and two for the second-degree parameters. The $\mathbf{M}_{3}^{\prime \prime}$ population adds no more than the $\mathrm{M}_{2}$ population for the first-degree parameters, but one new source i.e. $V_{\bar{M}_{3}^{\prime \prime}}$ for the second-degree ones. Neither the $\mathbf{M}_{3}^{\prime}$ nor $\mathbf{M}_{3}^{\prime \prime}$ populations alone can provide enough statistics, but they would greatly increase the reliability of the estimates, when grown together with the $\mathrm{M}_{3}$ population.

\section{(ii) Diallel crosses among $M_{2}$ plants}

The generalised diallel cross method of Dickinson and Jinks (1956) may be applicable to the $\mathrm{M}_{2}$ population of some plant species where crossing among individuals can be easily made. Substituting the $\mathrm{M}_{2}$ genotypic frequencies $1-\frac{3}{2} m, m$ and $\frac{1}{2} m$ for $\alpha, \beta$ and $\gamma$ of Dickinson and Jinks, and neglecting the terms with the second power of the mutation rate, we obtain the relationships,

and

$$
\bar{M}_{D}=\bar{M}_{0}-2[d]_{m}+2[h]_{m}
$$

$$
\begin{aligned}
V_{P_{1}} & =3 D_{m}+H_{m}-2 F_{m} \\
V_{P_{2}} & =3 D_{m}+\frac{1}{4} H_{m}-F_{m} \\
\bar{V}_{r} & =V_{r}=\frac{3}{4} D_{m}+\frac{3}{4} H_{m}-\frac{3}{2} F_{m}, \\
\bar{W}_{P_{1} / r} & =\frac{3}{2} D_{m}+\frac{1}{2} H_{m}-2 F_{m} \\
\bar{W}_{P_{2} / r} & =\frac{3}{2} D_{m}+\frac{1}{4} H_{m}-\frac{7}{4} F_{m} \\
W_{P_{1} / P_{2}} & =3 D_{m}+\frac{1}{2} H_{m}-\frac{3}{2} F_{m}
\end{aligned}
$$

where $\mathbf{M}_{D}$ stands for the overall mean of progeny families (see Dickinson and Jinks, 1956, for other symbols). Among these six statistics, the ones involving the parent $P_{1}$ should not be used for the reason given for the covariance between $M_{2}$ plants and their $M_{3}$ progenies.

This leaves one and three statistics for the estimation of the first- and second-degree parameters, respectively. From the latter, parameters $D_{m}$, 
$H_{m}$ and $F_{m}$ can therefore be estimated without any other populations. This may be the most advantageous features of the diallel cross method.

It should be noted, however, that the merit of diallel crosses is less than in the case of $F_{2}$ and later populations which have been made by artificial crossing between two different genotypes. Firstly, no parameters other than $D_{m}, H_{m}$ and $F_{m}$ are considered to be meaningful components of genetic variance. This lessens the amount of information from diallel crosses; for instance, the number of loci showing dominance as calculated by Dickinson and Jinks (1956) cannot be estimated. Secondly, the $V_{r}, W_{r}$ relationship cannot be used to detect failure of the simple additive-dominance model, because the values of $W_{r}-V_{r}$ differ with parental genotypes even if neither linkage disequilibrium nor non-allelic interaction exist. The geometrical interpretation of diallel crosses would be even more complicated in the presence of multiple allelism and variation due to chromosomal aberrations and cytoplasmic mutations.

\section{Interpretation of the Parameters}

The genetic nature and selection efficiency in later generations can be predicted using the estimates of the first- and second-degree parameters defined above. Table 3 gives the coefficients of the constituent parameters of the genetic variances and covariances in various generations. The use of the parameters is not confined to this.

Some rough information on the degree of dominance and gene distribution can be obtained by the use of the estimates. Supposing that each gene is alike in both genetic effect and mutation rate, the parameters may then be written as

$$
\begin{aligned}
{[d]_{m} } & =m k r_{1} d \\
{[h]_{m} } & =m k r_{2}|h| \\
D_{m} & =m k d^{2}, \\
H_{m} & =m k h^{2}, \\
F_{m} & =m k r_{3} d|h|,
\end{aligned}
$$

where the coefficient $r_{1}$ corresponds to the degree of gene association of Mather and Jinks (1971), which in this case assumes $+1,0$ and -1 according to whether all, half and none of the parental genes have larger contributions to the phenotype than mutant genes. The coefficient $r_{\mathbf{2}}$ measures the isodirectionality of dominance, taking the values of $+1,0$ and -1 if all, half and none of the alleles with increasing effect are dominant to the ones with decreasing effect, respectively. The coefficient $r_{3}$ shows the degree of association of dominant genes, the value of which is $+1,0$ and -1 when all, half and none of the parental alleles are dominant to mutant ones.

As in case of the populations obtained by crossing between two inbred lines, the ratio

gives the average dominance.

$$
\sqrt{\frac{H_{m}}{D_{m}}}=\frac{|h|}{d}
$$


The coefficients $r_{1}$ and $r_{2}$ cannot be estimated uniquely, but their signs, i.e. the signs of $[d]_{m}$ and $[h]_{m}$, together with the ratio

$$
\left(\frac{[h]_{m}}{\sqrt{H_{m}}}\right) /\left(\frac{[d]_{m}}{\sqrt{D_{m}}}\right)=\frac{r_{2}}{r_{1}}
$$

may in some cases give some idea on the balance sheet of gene association and isodirectionality of dominance. For instance, the situation of $[h]_{m}>0$ and $\left|r_{2} / r_{1}\right| \gg \mid$ strongly suggests that $r_{1} \approx 0$ and $r_{2} \approx 1$, namely, about half of the parental genes have increasing effect compared to mutant genes, dominance being positively directed for most of the genes concerned. The validity of this speculation would be confirmed if the value of $r_{3}$ calculated as

is around zero.

$$
\frac{F_{m}}{\sqrt{D_{m}} \cdot \overline{H_{m}}}=r_{3},
$$

Estimation of $m$ and $k$ has a great importance for both genetical and breeding research. There is no way of obtaining these values by the use of a single parental line. Theoretically, $m$ could be estimated if additional parameters were incorporated and estimated which included the second or higher power of $m$. To obtain reliable estimates of such parameters, however, unpracticably large-scale experiments with highly homogeneous environmental conditions would be required. The number $k$ cannot be estimated unless the values of $r_{1}$ and/or $r_{2}$ are obtained from some other source of information.

In the case where two parental lines with significantly different phenotypes, say $P_{1}$ and $P_{2}$, are available, the mutation rate can be estimated by

where

$$
\frac{[d]_{m 1}-[d]_{m 2}}{\bar{P}_{1}-\bar{P}_{2}}=\frac{2 \sum^{k^{\prime}} \pm m d}{2 \sum^{k^{\prime}} \pm d}=m,
$$

$\bar{P}_{1}$ and $\bar{P}_{2}=$ means of $P_{1}$ and $P_{2}$,

$[d]_{m 1}$ and $[d]_{m 2}=[d]_{m}$ of $p_{1}$ and $P_{2}$,

$k^{\prime}=$ number of genes for which the two parents carry different alleles.

Further, using the populations derived from hybridisation between the two parents, useful information on the ratio $k^{\prime} / k$ could be obtained by the relationship,

$$
\begin{gathered}
\frac{D}{D_{m}}=\frac{\sum^{k^{\prime}} d^{2}}{\sum m d^{2}}=\frac{k^{\prime} d^{2}}{k m d^{2}}, \\
\therefore \frac{k^{\prime}}{k}=\frac{m \cdot D}{D_{m}},
\end{gathered}
$$

where the parameter $D$ (variance component due to additive gene effect) is estimated by the method described in Mather and Jinks (1971). The calculation of the absolute value of $k$ is straightforward if two parental lines for which $k^{\prime}$ is known approximately are used. 


\section{Disqussion}

It has been assumed in deriving the formulae in previous sections that there are only two alleles for each gene, mutations occurring from one allele to the other. This may not be true in many cases. In the case of multiple allelism where parental genes mutate multidirectionally, the parameters must be somewhat differently defined.

If the original allele $G$ mutates to $r$ different alleles with mutation rates $m_{i}(i=1,2, \ldots, r)$, the mutation rate $m$ in the previously given formulae stands for the total mutation rate summed over all of the different mutant

alleles, i.e. $\sum^{r} m_{i}$. The genetic effect $d$ now becomes half the difference between parental genotypic value and weighted average of the $r$ different mutant homozygotes, the dominance effect $h$ being measured by the deviation from the mid-point of these two values.

Mathematical formulation of the genetic parameters need not be changed by this generalisation, but interpretation of them must be modified according to the above redefinition. The estimation of the mutation rate $m$ and the ratio $k^{\prime} / k$ as described in the preceding section is invalid in this case, because the value of $d$ in mutagenically treated populations may be different from that of the populations derived from hybridisation between inbred lines.

Extragenic variations due to chromosomal aberrations and cytoplasmic mutations impose complications on the interpretation of the parameter estimates. The hereditary behaviour of extragenic mutations and their genetic contributions to quantitative characters have not yet been well defined, and therefore, no biometrical formulation can be made to separate extragenic components from other genetic variations. Following Oka et al. (1958), one way to counter this would be to remove so-called macro-mutants from the population to be analysed, since most macro- or visible mutants for morphological and physiological characters are expected to arise from chromosomal, cytoplasmic, or major gene mutations.

This method, however, is by no means perfect. The $M_{n}$ populations seldom show clearly discontinuous distribution, and therefore the distinction of macro-mutants has been arbitrary. In addition, some extragenic mutations may have relatively small genetic effects. The fact that aberrant mutants such as steriles, dwarfs and chlorophyll deficients often segregate in the progeny of seemingly normal plants (e.g. Yamagata and Syakudo, 1963) suggests that some chromosomal mutations are partly or completely recessive. About half of such mutations will remain uneliminated each generation, even if they are unambiguously removed in the homozygous state. To minimise the influence of extragenic mutations, late generations should be used, taking the greatest care to minimise the bias in gene frequency due to natural selection and random drift during generation advance. Comparison of the parameter estimates for early and late generations would give some useful information on the nature of non-genic variations.

Epistatic gene interaction is another factor causing a great complication in interpretation, although its contribution to the total variation is in general not very large. Methods for detecting epistatic variation in induced variants may be developed based on the principle adopted by Perkins and Jinks (1970), which will be discussed elsewhere. 
Heritabilities have often been calculated to predict the efficiency of selection on induced continuous variation (e.g. Rao and Siddig, 1976). The predictive power of these heritabilities, however, has not been satisfactory in general; large differences exist between expected and realised heritabilities, the latter being much smaller than the former in most cases. This may be explained partly by dominance and epistatic gene actions and by unpredictable genotype $\times$ environment interaction. But, the main reason seems to be ascribable to inadequate design of experiment.

Control or non-treated populations have not been used at all, or used only on a much smaller scale than mutagenically treated populations. This inevitably causes overestimation of some genetic variance components, since environmental variances such as between-plot variance cannot be unambiguously separated. Heritability estimates will be more or less inflated if an overestimated variance component is used as a numerator in calculating heritability.

Apart from the various sources of experimental error, heritability should be equal or sufficiently close to a regression coefficient of certain pedigree units (individuals, lines etc.) on their progeny means, if it is to be used as a predictor of the genetic improvement achieveable by each cycle of artificial selection. In most cases in mutation researches, heritabilities have been calculated as the ratios of genetic variance components of certain pedigree units to the total phenotypic variance. Heritabilities of this type may be useful for measuring the relative contribution of each genetic variance component to the whole and for identifying the major sources of the genetic variation, but they do not meet the above requirement. Estimates of the parameters $D_{m}, H_{m}$ and $F_{m}$ together with appropriate environmental variance components allow us to calculate any type of heritability for any generation.

Acknoweledgments. - The author is greatly indebted to Professor J. L. Jinks for his valuable discussion and suggestions. He also thanks Drs M. J. Kearsey and H. S. Pooni for their critical reading of the manuscript. This work was carried out while the author was staying at the Department of Genetics of the University of Birmingham under the sponsorship of the British Council.

\section{REFERENGES}

AAstvert, K., AND GAUL, H. 1967. Variation and selection of micro-mutants. Radiation Botany, 7, 353-361.

Dickinson, A. G., AND JINKs, J. L. 1956. A generalised analysis of diallel crosses. Genetics, 41, 65-78.

HAYMAN, B. I. 1960. Maximum likelihood estimation of genetic components of variation. Biometrics, 16, 369-381.

JNKs, J. L. 1954. The analysis of continuous variation in a diallel cross of Nicotiana rustica varieties. Genetics, 39, 767-788.

LAWRENGE, C. W. 1965. Radiation induced polygenic mutation. The Use of Induced Mutations in Plant Breeding. Radiation Botany 5 (Suppl.), 491-496.

MATHER, K. 1949. Biometrical Genetics. Methuen, London.

mather, K., AND JiNks, J. L. 1971. Biometrical Genetics, 2nd edition. Chapman and Hall, London.

OKA, H., HAYASH, J., AND HANWAY, D. G. 1958 . Induced mutation of polygenes for quantitative characters in rice. 7 . Herod., 49, 11-14.

osone, к. 1963. On the development mechanism of mutated cells. Recent Advance in Breeding, 4, 79-87.

PERKINS, J. M., AND JINKs, J. L. 1970. Detection and estimation of genotype-environmental, linkage and epistatic components of variation for a metrical trait. Heredity, 25, 157-177. 
RAO, G. M., AND sIDDIQ, E. A. 1976. Studies on induced variability for amylose content with reference to yield components and protein characteristics in rice. Environmental and Experimental Botany, 16, 177-188.

SARVELIA, P., NIRAN, R. A., AND KONZAK, C. F. 1962. Relation of embryo structure, node position, tillering and depth of planting to the effects of X-rays in barley. Radiation Botany, 2, 89-108.

VIRK, D. s., JINKS, J. L., AND POONI, H. s. 1978. The assessment of induced continuous variation in pure-breeding lines following selfing. Heredity, 40, 255-268.

YAMAGATA, H., AND SYAKUDO, K. 1963. Studies on the utility of artificial mutations in plant breeding. II. On the appearance of mutations in the $\mathrm{X}_{3}$ generation following $\gamma$-ray irradiation of rice seeds. Fapan. F. Breed., 13, 14-20.

YONEZAWA, K. 1975. Method and efficiency of mutation breeding for quantitative characters. Gamma Field Symposia, 14, 39-58.

YONEZAWA, K., AND YAMAGATA, $\mathrm{H}$. 1975. Comparison of the scoring methods for mutation frequency in self-pollinating disomic plants. Radiation Botany, 15, 241-256.

YONEZAWA, K., AND YAMAGATA, H. 1977. On the optimum mutation rate and optimum mutagen dose for practical mutation breeding. Euphytica, 26, 413-426. 